\title{
Theta Term in QCD sum rules at Finite Temperature and the Neutron Electric Dipole Moment
}

\author{
Mohamed Chabab \\ LPHEA, Physics Department, Faculty of Science-Semlalia, \\ P.O. Box 2390, Marrakesh, Morocco \\ mchabab@ucam.ac.ma
}

\begin{abstract}
By using thermal QCD sum rules to investigate the $\bar{\theta}$ induced neutron electric dipole moment $d_{n}$, we have examined the behaviour of broken $\mathrm{CP}$ symmetry at finite temperature. We find that, below the critical temperature, the ratio $\left|\frac{d_{n}}{\theta}\right|$ slightly decreases but survives at temperature effects, implying T nonrestoration of CP-invariance [1].
\end{abstract}

\section{INTRODUCTION}

To establish connection between particle physics and cosmology, it is essential to study the behaviour of symmetry breaking in the early universe, i.e. at high temperature. The CP symmetry is, without doubt, one of the most fundamental symmetries in nature. It is intimately related to theories of interactions between elementary particles and represents a cornerstone in constructing grand unified and supersymmetric models. However, its breaking still carries a cloud of mystery in particle physics and cosmology since it is necessary to explain baryogenesis and is required by theories with domain walls.

According to the $\mathrm{CPT}$ theorem, $\mathrm{CP}$ violation implies $\mathrm{T}$ violation. The latter is tested through the measurement of the neutron electric dipole moment (NEDM) $d_{n}$. The upper experimental limit gives confidence that the NEDM can be another manifestation of CP breaking. In the Standard Model, CP violation is originated from two sources: the first source, which appears in the electroweak sector, is parametrized by a single phase in the Cabbibo-Kobayashi-Maskawa (CKM) quark 
mixing matrix [2]. The other source is due to the the so called $\theta$-term of QCD. In fact, the the QCD effective lagrangian contains an additional CP-odd four dimensional operator embedded in the following topological term:

$$
L_{\theta}=\theta \frac{\alpha_{s}}{8 \pi} G_{\mu \nu} \tilde{G}^{\mu \nu},
$$

where $G_{\mu \nu}$ is the gluonic field strength, $\tilde{G}^{\mu \nu}$ is its dual and $\alpha_{s}$ is the strong coupling constant. The $G_{\mu \nu} \tilde{G}^{\mu \nu}$ quantity is a total derivative, consequently it contribute to the physical observables only through non perturbative effects. The NEDM is related to the $\bar{\theta}$-angle by the following relation:

$$
d_{n} \sim \frac{e}{M_{n}}\left(\frac{m_{q}}{M_{n}}\right) \bar{\theta} \sim\left\{\begin{array}{l}
2.7 \times 10^{-16} \bar{\theta} \\
5.2 \times 10^{-16} \bar{\theta}
\end{array}\right. \text { 这 }
$$

and consequently, according to the experimental measurements $d_{n}<1.1 \times$ $10^{-25} \mathrm{ecm}$ [5], the $\bar{\theta}$ parameter must be less than $2 \times 10^{-10}$ [6]. The well known strong CP problem consists in explaining the smallness of $\bar{\theta}$. In this regard, several scenarios were suggested. The most popular one is the Peccei and Quinn [7], in which $\bar{\theta}]$ is identified to a very light pseudo goldstone boson called the axion. This particle arises from the spontaneous breakdown of a global $U_{A}(1)$ symmetry and may well be important to explain the puzzle of dark matter providing a peace of information on the missing mass of the universe [8].

Our aim in this work is to study the thermal behaviour of the CP symmetry breaking and the temperature effects on its restoration. This is motivated by the possibility to restore some broken symmetries by increasing the temperature.

This paper is organized as follows: Section 2 is devoted to the calculations of the NEDM induced by the $\bar{\theta}$ using QCD sum rules. In section 3, we show how one introduces temperature in QCD sum rules calculations. The last section is devoted to a discussion and qualitative analysis of the thermal effects on the CP symmetry.

\section{NEDM from QCD sum rules}

Since the NEDM has essaentially a non perturbative nature, one should any how take into account some effects which escape the perturbative treatement. One such approach, dealing with the strong strong coupling regime and based on first principales of the theory is QCD sum rules. Such approach has been applied successfully to the investigation of hadronic properties at low energies, particularly to certain baryonic magnetic form factor. In order to derive the NEDM through through this approach [9, 10], we consider a lagrangian containing the following P 
and $\mathrm{CP}$ violating operators:

$$
L_{P, C P}=-\theta_{q} m_{*} \sum_{f} \bar{q}_{f} i \gamma_{5} q_{f}+\theta \frac{\alpha_{s}}{8 \pi} G_{\mu \nu} \tilde{G}^{\mu \nu} .
$$

$\theta_{q}$ and $\theta$ are respectively two angles coming from the chiral and the topological terms and $m_{*}$ is the quark reduced mass given by $m_{*}=\frac{m_{u} m_{d}}{m_{u}+m_{d}}$. Then, as usual, we start from the two points correlation function in QCD background with a nonvanishing $\theta$ and in the presence of a constant external electomagnetic field $F^{\mu \nu}$ [10]:

$$
\Pi\left(q^{2}\right)=i \int d^{4} x e^{i q x}<0|T\{\eta(x) \bar{\eta}(0)\}| 0>_{\theta, F} .
$$

where $\eta(x)$ is the neutron interpolating current [12]:

$$
\eta=2 \epsilon_{a b c}\left\{\left(d_{a}^{T} C \gamma_{5} u_{b}\right) d_{c}+\beta\left(d_{a}^{T} C u_{b}\right) \gamma_{5} d_{c}\right\}
$$

and $\beta$ is a mixing parameter. To select the appropriate Lorentz stucture, $\Pi\left(q^{2}\right)$ is expanded in terms of the electromagnetic charge as:

$$
\Pi\left(q^{2}\right)=\Pi^{(0)}\left(q^{2}\right)+e \Pi^{(1)}\left(q^{2}, F^{\mu \nu}\right)+O\left(e^{2}\right) .
$$

The first term $\Pi^{(0)}\left(q^{2}\right)$ is the nucleon propagator which includes only the CP-even parameters, while the second term $\Pi^{(1)}\left(q^{2}, F^{\mu \nu}\right)$ is the polarization tensor which may be expanded through Wilson OPE as: $\sum C_{n}<0|\bar{q} \Gamma q| 0>_{\theta, F}$, where $\Gamma$ is an arbitrary Lorentz structure and $C_{n}$ are the Wilson coefficient functions calculable in perturbation theory [14, 13]. From this expansion, we keep only the CP-odd contribution part. The electromagnetic dependence of these matrix elements is determined in terms of the magnetic susceptibilities $\kappa, \chi$ and $\xi$, defined as [13]:

$$
\begin{aligned}
<0\left|\bar{q} \sigma^{\mu \nu} q\right| 0>_{F} & =\chi e_{q} F^{\mu \nu}<0|\bar{q} q| 0> \\
g<0\left|\bar{q} G^{\mu \nu} q\right| 0>_{F} & =\kappa e_{q} F^{\mu \nu}<0|\bar{q} q| 0> \\
2 g<0\left|\bar{q} \tilde{G}^{\mu \nu} q\right| 0>_{F} & =\xi e_{q} F^{\mu \nu}<0|\bar{q} q| 0>
\end{aligned}
$$

Besides, by considering the anomalous axial current, one obtains the following $\theta$ dependence of $<0|\bar{q} \Gamma q| 0>_{\theta}$ matrix elements [9]:

$$
m_{q}<0|\bar{q} \Gamma q| 0>_{\theta}=i m_{*} \theta<0|\bar{q} \Gamma q| 0>+O\left(m_{q}^{2}\right)
$$

where $m_{q}$ and $m_{*}$ are respectively the quark and reduced masses. $O\left(m_{q}^{2}\right)$ connection is negligible since $m_{\eta}>>m_{\pi}$.

Putting altogether the above ingredients and after a straightforward calculation [10], the following expression of $\Pi^{(1)}\left(q^{2}, F^{\mu \nu}\right)$ for the neutron is derived: 


$$
\begin{aligned}
\Pi\left(-q^{2}\right)= & -\frac{\bar{\theta} m_{*}}{64 \pi^{2}}<0|\bar{q} q| 0>\{\tilde{F} \sigma, \hat{q}\}\left[\chi(\beta+1)^{2}\left(4 e_{d}-e_{u}\right) \ln \left(\frac{\Lambda^{2}}{-q^{2}}\right)\right. \\
& -4(\beta-1)^{2} e_{d}\left(1+\frac{1}{4}(2 \kappa+\xi)\right)\left(\ln \left(\frac{-q^{2}}{\mu_{I R}^{2}}\right)-1\right) \frac{1}{-q^{2}} \\
& \left.-\frac{\xi}{2}\left(\left(4 \beta^{2}-4 \beta+2\right) e_{d}+\left(3 \beta^{2}+2 \beta+1\right) e_{u}\right) \frac{1}{-q^{2}} \ldots\right],
\end{aligned}
$$

where $\bar{\theta}=\theta+\theta_{q}$ is the physical phase and $\hat{q}=q_{\mu} \gamma^{\mu}$.

The QCD expression (2.7) is confronted to the phenomenological parametrisation $\Pi^{\text {Phen }}\left(-q^{2}\right)$ written in terms of the Neutron hadronic properties. The latter is given by:

$$
\Pi^{\text {Phen }}\left(-q^{2}\right)=\{\tilde{F} \sigma, \hat{q}\}\left(\frac{\lambda^{2} d_{n} m_{n}}{\left(q^{2}-m_{n}^{2}\right)^{2}}+\frac{A}{\left(q^{2}-m_{n}^{2}\right)}+\ldots\right)
$$

where $m_{n}$ is the neutron mass, $e_{q}$ is the quark charge. A and $\lambda^{2}$, which originate from the phenomenological side of the sum rule, represent respectively a constant of dimension 2 and the neutron coupling constant to the interpolating current $\eta(x)$. This coupling is defined via a spinor $v$ as $<0|\eta(x)| n>=\lambda v e^{\alpha \gamma_{5}}$.

\section{Thermal NEDM sum rules}

The introduction of finite temperature effects may provide more precision to the phenomenological values of hadronic observables. Within the framework of QCD sum rules, the Temperature evolution of the correlation functions manifests itself in the thermal average of the Wilson operator expansion [15, 18]. Hence, at relatively low temperature, the system can be regarded as a non interacting gas of bosons. In this approximatyion, the Thermal dependance of the vacuum condensates can be written as :

$$
<O^{i}>_{T}=<O^{i}>+\int \frac{d^{3} p}{2 \epsilon(2 \pi)^{3}}<\pi(p)\left|O^{i}\right| \pi(p)>n_{B}\left(\frac{\epsilon}{T}\right)
$$

where $\epsilon=\sqrt{p^{2}+m_{\pi}^{2}}, n_{B}=\frac{1}{e^{x}-1}$ is the Bose-Einstein distribution and $\left\langle O^{i}\right\rangle$ is the standard vacuum condensate (i.e. at $\mathrm{T}=0$ ). In this approximation, we only kept the pion contributions, since in the low temperature region, the effects of heavier resonances $(\Gamma=K, \eta,$. etc $)$ are dumped by their distibution functions $\sim e^{\frac{-m_{\Gamma}}{T}}[17$. To compute the pion matrix elements, we apply the soft pion theorem given by:

$$
<\pi(p)\left|O^{i}\right| \pi(p)>=-\frac{1}{f_{\pi}^{2}}<0\left|\left[Q_{5}^{a},\left[Q_{5}^{a}, O^{i}\right]\right]\right| 0>+O\left(\frac{m_{\pi}^{2}}{\Lambda^{2}}\right)
$$


where $\Lambda$ is a hadron scale and $Q_{5}^{a}$ is the isovector axial charge defined by:

$$
Q_{5}^{a}=\int d^{3} x \bar{q}(x) \gamma_{0} \gamma_{5} \frac{\tau^{a}}{2} q(x)
$$

Direct application of the above formula to the quark and gluon condensates shows the following features 16, 17]:

(i) Only $<\bar{q} q>$ is sensitive to temperature. Its behaviour at finite $\mathrm{T}$ is given by:

$$
<\bar{q} q>_{T} \simeq\left(1-\frac{\varphi(T)}{8}\right)<\bar{q} q>
$$

where $\varphi(T)=\frac{T^{2}}{f_{\pi}^{2}} B\left(\frac{m_{\pi}}{T}\right)$ with $B(z)=\frac{6}{\pi^{2}} \int_{z}^{\infty} d y \frac{\sqrt{y^{2}-z^{2}}}{e^{y}-1}$ and $f_{\pi}$ is the pion decay constant $\left(f_{\pi} \simeq 93 \mathrm{MeV}\right)$. The variation with temperature of the quark condensate $<\bar{q} q>_{T}$ results in two different asymptotic behaviours, namely:

$$
\begin{gathered}
<\bar{q} q>_{T} \simeq\left(1-\frac{T^{2}}{8 f_{\pi}^{2}}\right)<\bar{q} q>\quad \text { for } \quad \frac{m_{\pi}}{T} \ll 1 \\
<\bar{q} q>_{T} \simeq\left(1-\sqrt{\frac{\pi m_{\pi}}{2 T} \frac{T^{2}}{8 f_{\pi}^{2}} e^{\frac{-m_{\pi}}{T}}}\right)<\bar{q} q>\quad \text { for } \quad \frac{m_{\pi}}{T} \gg 1
\end{gathered}
$$

(ii) The gluon condensate is nearly constant at low temperature and a $\mathrm{T}$ dependence occurs only at order $T^{8}$.

The determination of the ratio $\frac{d_{n}}{\theta}$ sum rules at non zero temperature is now easily performed by applying Borel operator to both parametrisation of the Neutron correlation function shown in Eqs. (2.7) and (2.8). Then finite temperature effects are introduced via the procedure discussed above. Finally, by invoking the quarkhadron duality, we deduce the final sum rules of the $\bar{\theta}$ induced NEDM at finite temperature:

$\frac{d_{n}}{\bar{\theta}}(T)=-\frac{M^{2} m_{*}}{16 \pi^{2}} \frac{1}{\lambda_{n}^{2}(T) M_{n}(T)}\left(1-\frac{\varphi(T)}{8}\right)<\bar{q} q>\left[4 \chi\left(4 e_{u}-e_{d}\right)-\frac{\xi}{2 M^{2}}\left(4 e_{u}+8 e_{d}\right)\right] e^{\frac{M_{n}^{2}}{M^{2}}}$

where $\mathrm{M}$ represents the Borel parameter. The single pole contribution entering the sum rules via the constant A has been neglected, as suggested in [9].

The value of $\beta$ has been set to 1 in (3.5). This choice is more appropriate for us since it suppresses the infrared divergences. The T-evolution of the coupling constant and the mass of the neutron were determined from the thermal nucleon sum rules [17].

Within the dilute pion gas approximation, Eletsky has shown that the contribution induced the pion-nucleon scattering has to be considered 19. It enters the nucleon sum rules through the coupling constant $g_{\pi N N}$, whose values lie within the 
range 13.5-14.3 [20].

Numerical analysis is performed with the following input parameters: the Borel mass has been chosen within the values $M^{2}=0.55-0.7 \mathrm{GeV}^{2}$ which correspond to the optimal range (Borel window) in the $\frac{d_{n}}{\theta}$ sum rule at $T=0$ [10]. For the $\chi$ and $\xi$ susceptibilities we take $\chi=-5.7 \pm 0.6 \mathrm{GeV}^{-2}$ [21] and $\xi=-0.74 \pm 0.2$ [22]. As to the vacuum condensates appearing in (3.5), we use their standard values [1].

\section{Analysis and Conclusion}

We have established the relation between the NEDM and $\bar{\theta}$ angle at non zero temperature from QCD sum rules. We find that the behaviour of the ratio $\frac{d_{n}}{\theta}$ is connected to the thermal evolution of the pion parameters $f_{\pi}, m_{\pi}$ and of $g_{\pi N N}$.

By analysing the ratio as a function of $\mathrm{T}$ in the region of validity of thermal sumrules $\left[0, T_{c}\right]$, we learn that $\left|\frac{d_{n}}{\theta}\right|$ decreases smoothly with $\mathrm{T}$ (about $16 \%$ variation for temperature values up to $200 \mathrm{MeV}$ ) but survives at finite temperature. This means that either the NEDM value decreases or $\bar{\theta}$ increases. Consequently, for a fixed value of $\bar{\theta}$ the NEDM decreases but it does not exhibit any critical behaviour. Furthermore, if we start from a non vanishing $\bar{\theta}$ value at $T=0$, it is not possible to remove it at finite temperature. We also note that $\left|\frac{d_{n}}{\theta}\right|$ grows as $M^{2}$ or $\chi$ susceptibility increases. It also grows with quark condensate rising. However this ratio is insensitive to both the $\xi$ susceptibility and the coupling constant $g_{\pi N N}$. We notice that for higher temperatures, the analysis of $\left|\frac{d_{n}}{\theta}\right|=f\left(\frac{T}{T_{c}}\right)$ exhibits a brutal increase justified by the fact that for temperatures beyond the critical value $T_{c}$, at which the chiral symmetry is restored, the constants $f_{\pi}$ and $g_{\pi N N}$ become zero and consequently from $\operatorname{Eq}(3.5)$ the ratio $\frac{d_{n}}{\theta}$ behaves as a non vanishing constant. The large difference between the values of the ratio for $T<T_{c}$ and $T>T_{c}$ may be a consequence of the fact that other contributions to the the spectral function have ben neglected, like the scattering process $N+\pi \rightarrow \Delta$. These contributions which are of the order $T^{4}$, are negligible in the low temperture region but become substantial for $T \geq T_{c}$. Moreover, this difference may also originate from the use of soft pion approximation which is valid essentially for low $T\left(T<T_{c}\right)$. Therefore it is clear from this qualitative analysis, which is based on the soft pion approximation, that temperature does not play a fundamental role in the suppression of the undesired $\theta$-term and hence the broken CP symmetry is not restored [24]. Indeed, some exact symmetries can be broken by increasing temperature [23, 24]. The symmetry non restoration phenomenon, which means that a broken symmetry at $\mathrm{T}=0$ remains broken even at high temperature, is essential for discrete symmetries, CP symmetry in particular. Indeed, the symmetry non restoration allows us to avoid wall domains inherited after the phase transition [25] and to explain the baryogenesis phenomenon in cosmology [26]. Furthermore, it can be very useful for solving the monopole problem in grand unified theories [27. 


\section{AKNOWLEDGMENTS}

The author is deeply grateful to Prof. Brigitte Hiller and to the organization Committe for the invitation to the Second International Conference on Hadron Physics. He also wishes to thank Prof. Joao Providencia for his hospitality during the visit to CPT at Coimbra.

This work is supported by the convention de cooperation between CNRST/ICCTI 681.02/CNR.

\section{References}

[1] Chabab, M., El Biaze, N., and Markazi, R., J. Phys. G27, 2275 (2001).

[2] Cabibbo, N., Phys. Rev. Lett. 10, 531 (1963);

Kobayashi, M and Maskawa, T., Prog. Theor. Phys. 49, 652 (1973).

[3] Baluni, V, Phys. Rev D19, 2227 (1979).

[4] Crewther, R., Di Vecchia, P., Veneziano, G. and Witten, E., Phys. Letters B88, 123 (1979).

[5] Barnett, R. M., and al, Phys. Rev. D54, 1 (1996).

[6] Peccei, R. D., hep-ph/9807516.

[7] Peccei, R. D., and Quinn, H. R., Phys. Rev. D16, 1791 (1977).

[8] Lazarides, G., and Shafi, Q., "Monopoles, Axions and Intermediate Mass Dark Matter", hep-ph/0006202.

[9] Pospelov, M. and Ritz, A., Nucl. Phys. B558, 243 (1999).

[10] Pospelov, M., and Ritz, A., Phys. Rev. Letters 83, 2526 (1999).

[11] Shifman, M. A., Vainshtein, A. I., and Zakharov, V. I., Nucl. Phys. B147, 385 (1979).

[12] Ioffe, B. L., Nucl. Phys. B188, 317 (1981);

Chung, Y. and al, Phys. Lett.B102, 175 (1981); Nucl. Phys. B197, 55 (1982)

[13] Ioffe, B. L., and Smilga, A. V., Nucl. Phys. B232, 109 (1984).

[14] Shifman, M.A., Vainshtein, A. I., and Zakharov, V. I., Nucl. Phys. B166, 493 (1980). 
[15] Bochkarev, A. I., and Shaposhnikov, M. E., Nucl. Phys. B268, 220 (1986).

[16] Gasser, J., and Leutwyler, H., Phys. Letters B184, 83 (1987);

Leutwyler, H., in $Q C D 20$ years later, edited by . P.M. Zerwas and H.A. Kastrup, World scientific Proceedings, Singapore, 1992. 1993).

[17] Adami, C., and Zahed, I., Phys.Rev D45, 4312 (1992);

Hatsuda, T., Koike, Y., and Lee, S. H., Nucl. Phys. B394, 221 (1993).

[18] Mallik, S., and Mukherjee, K., Phys. Rev. D58, 096011 (1998).

[19] Eletsky, V. L., Phys. Lett. B245, 229 (1990); Phys. Letters B352, 440 (1995)

[20] Blomgten, J., in A critical issue in the determination of the pion nucleon decay constant, Phys. Scripta T87, 53 (2000).

[21] Belyaev, V. M., and Kogan, Y. I., Sov. J. Nucl. Phys. 40, 659 (1984).

[22] Kogan, I. I., and Wyler, D., Phys. Letters B274, 100 (1992).

[23] Weinberg, S., Phys. Rev D9, 3357 (1974).

[24] Mohapatra, R. N., and Senjanovic, G., Phys. Rev. D20, 3390 (1979) ; Dvali, G., Melfo, A., and Senjanovic, G., Phys.Rev. D54, 7857 (1996).

[25] Zeldovich, Y. B., Kobzarev, I. Y., and Okun, L., JETP. 40, 1 (1974);

Kibble, T. W., J. Phys. A9, 1987 (1976); Phys. Rep. 67, 183 (1980).

[26] Sakharov, A., JETP Letters 5, 24 (1967).

[27] Dvali, G., Melfo, A., and Senjanovic, G., Phys. Rev. Letters 75, 4559 (1995). 